\title{
Retroperitoneoscopic approach for urolithiasis treatment
}

\author{
Jose Luis Bauza ${ }^{1}$, Valentí Tubau ${ }^{1}$, Javier Brugarolas ${ }^{1}$, Luis Ladaria ${ }^{1}$, Carlos Aliaga ${ }^{1}$, Pedro Piza ${ }^{1}$, \\ Enrique Pieras ${ }^{1}$
}

${ }^{1}$ Department of Urology, Hospital Universitario Son Espases, Palma de Mallorca, Illes Balears, Spain

\section{ABSTRACT}

Objective: To show the main indications of retroperitoneoscopy (RP) for the treatment of urolithiasis. The use of RP approach has been limited, being narrow working space the major issue to overcome (1), especially in non-expert hands. However, RP has the added advantages of no peritoneal contamination, a quick recovery of bowel function (2) and the possibility to use it in combination with other endourological techniques (3) and innovative technology.

Materials and Methods: We performed a retrospective analysis of 22 patients treated by the retroperitoneoscopic approach due to urolithiasis disease between 2015-2017. Type of surgery, stone free rate (SFR), complications according to Clavien-Dindo classification and mean hospital stay were recorded. Radical and partial nephrectomy cases were excluded for the SFR calculation. Descriptive statistical analysis was done using SPSS v21.

Results: Of the 22 patients treated by the retroperitoneoscopic approach, 9 underwent a ureterolithotomy, 4 underwent a nephrolithotomy, 8 were nephrectomies and 1 was a polar nephrectomy. In 3 cases we used the indocianine green fluorescence (ICG) to find avascular planes, reduce the bleeding, permitting enhanced visualization and reconstruction. In 3 cases an additional percutaneous approach was used, increasing the SFR chances. Eleven of thirteen (84.6\%) patients were stone free following the procedure. Tree complications were recorded, two Clavien II and one Clavien III complications. Mean hospital stay was 4 days.

Conclusions: Retroperitoneoscopic approach is a good alternative for the treatment of large impacted ureteral stones, large pielic stones and for non-functional kidney removal due to stone disease. In expert hands, it can be safely used with a good SFR. The combination with ICG or other endourological techniques is feasible, allowing higher SFR.

\section{REFERENCES}

1. Won YS, Lee SJ, Kim HY, Lee DS. Five-Year Single Center Experience for Retroperitoneoscopic Ureterolithotomy. J Laparoendosc Adv Surg Tech A. 2017;27:128-33.

2. Chipde SS, Agrawal S. Retroperitoneoscopic pyelolithotomy: a minimally invasive alternative for the management of large renal pelvic stone. Int Braz J Urol. 2014;40:123-4;discussion 124.
3. Tepeler A, Akman T, Tok A, Kaba M, Binbay M, Müslümano lu AY, et al. Retroperitoneoscopic nephrectomy for non-functioning kidneys related to renal stone disease. Urol Res. 2012;40:559-65.

\section{CONFLICT OF INTEREST}

None declared. 


\section{ARTICLE INFO}

\section{Jose Luis Bauza Quetglas}

http://orcid.org/0000-0002-8955-483X

Available at: http://www.intbrazjurol.com.br/video-section/20190099_Bauza_et_al

Int Braz J Urol. 2020; 46 (Video \#17): 678-9

Submitted for publication:

February 11, 2019

Accepted after revision:

May 31, 2019

Published as Ahead of Print:

August 10, 2019
Correspondence address: Jose Luis Bauza Quetglas, MD Department of Urology, Hospital Universitario Son Espases

79 Valldemossa Rd

Palma de Mallorca, 70120, Spain Telephone: + 34608 688-560 E-mail: peplluis15@hotmail.com 